\title{
ON FOUNDATIONS OF THE CONLEY INDEX THEORY
}

\author{
ROMAN SRZEDNICKI \\ Institute of Mathematics, Jagiellonian University \\ Reymonta 4, 30-059 Kraków, Poland \\ E-mail: srzednic@im.uj.edu.pl
}

1. Introduction. The Conley index theory was introduced by Charles C. Conley (1933-1984) in [C1] and a major part of the foundations of the theory was developed in $\mathrm{Ph}$. D. theses of his students, see for example [Ch, Ku, Mon]. The Conley index associates the homotopy type of some pointed space to an isolated invariant set of a flow, just as the fixed point index associates an integer number to an isolated set of fixed points of a continuous map. Examples of isolated invariant sets arise naturally in the critical point theory - each isolated critical point of a functional is also an isolated invariant set of its gradient flow. If the critical point is nondegenerate then its Conley index is equal to the homotopy type of the pointed $k$-sphere, where $k$ is the Morse index of that point. There are other relations to Morse theory, for example a generalization of Morse inequalities can be achieved.

The aim of this note is to describe briefly some basic facts of the Conley index theory for (continuous-time) flows. We refer to [C2, Ry, S1, Smo] for a more detailed presentation. We do not touch more advanced topics of the theory: the Conley index as a connected simple system (see [C2, Ku, McM, S1]), connection and transition matrices (see [F1, F2, FM, McM, Mi1, Moe, Re]), infinite dimensional Conley indices (see [Be, Ry], the Conley index for multivalued flows (see [KM, Mr2]), Conley-type indices for discrete-time flows (see [Mr3, RS, Sz]), equivariant Conley indices (see [Ba, Ge]), and relations to the Floer homology (see [S2]). (The list of bibliography items is far from completeness.) Moreover, we do not present any applications of the index. For some more recent results we refer to [Mi2]. We also refer to the other articles in this Proceedings.

2. Topological background. In this section $X$ denotes an arbitrary topological space. Let $A \subset X$. Assume that $A$ is nonempty. Then $X / A$ is defined as the quotient

1991 Mathematics Subject Classification: Primary 54H20; Secondary 34C35, 58E05, 58F25.

Supported by KBN, Grant 2 P03A 04010.

The paper is in final form and no version of it will be published elsewhere. 
space obtained by identifying all points of $A$. The point in $X / A$ obtained by collapsing of $A$ is denoted by $[A]$. In order to define $X / A$ in the case $A=\emptyset$ we fix a point $*$ - we treat it as a point outside of any set considered, define $X / \emptyset:=X \cup\{*\}$, and put $[\emptyset]:=*$.

Let $x_{0} \in X$. A pair $\left(X, x_{0}\right)$ is called a pointed topological space (or a space with a base point). In particular, the pair $(X / A,[A])$ is an example of a pointed space even if $X$ or $A$ is empty. Let $\left(Y, y_{0}\right)$ be another pointed space. We write $f:\left(X, x_{0}\right) \rightarrow\left(Y, y_{0}\right)$ provided $f: X \rightarrow Y$ is continuous and $f\left(x_{0}\right)=y_{0}$. If $g:\left(X, x_{0}\right) \rightarrow\left(Y, y_{0}\right)$ then $f \simeq g$ rel $x_{0}$ means the existence of a continuous map $F: X \times[0,1] \rightarrow Y$ such that $F(\cdot, 0)=f, F(\cdot, 1)=g$, and $F\left(x_{0}, t\right)=y_{0}$ for every $t \in[0,1]$. We define

$$
\begin{aligned}
& \left(X, x_{0}\right) \vee\left(Y, y_{0}\right):=\left(X \times\left\{y_{0}\right\} \cup\left\{x_{0}\right\} \times Y,\left(x_{0}, y_{0}\right)\right), \\
& \left(X, x_{0}\right) \wedge\left(Y, y_{0}\right):=(X \times Y / X \vee Y,[X \vee Y]) .
\end{aligned}
$$

We say that $\left(X, x_{0}\right)$ and $\left(Y, y_{0}\right)$ have the same pointed homotopy type provided there exist maps $f:\left(X, x_{0}\right) \rightarrow\left(Y, y_{0}\right)$ and $g:\left(Y, y_{0}\right) \rightarrow\left(X, x_{0}\right)$ such that $g \circ f \simeq \operatorname{id} \operatorname{rel}_{0}$ and $f \circ g \simeq \operatorname{id}_{Y}$ rel $y_{0}$. The pointed homotopy type of $\left(X, x_{0}\right)$ is denoted by $\left[X, x_{0}\right]$. We define the trivial pointed homotopy type as $\overline{0}:=[\{*\}, *]$. One can check correctness of the following definitions:

$$
\begin{aligned}
& {\left[X, x_{0}\right] \vee\left[Y, y_{0}\right]:=\left[\left(X, x_{0}\right) \vee\left(Y, y_{0}\right)\right],} \\
& {\left[X, x_{0}\right] \wedge\left[Y, y_{0}\right]:=\left[\left(X, x_{0}\right) \wedge\left(Y, y_{0}\right)\right] .}
\end{aligned}
$$

By $\Sigma^{k}$ we denote $\left[S^{k}, s_{0}\right]$ and by $\Pi^{k}$ we denote $\left[\mathbb{R} P^{k}, p_{0}\right]$, where $S^{k}$ and $\mathbb{R} P^{k}$ denote the $k$-dimensional sphere and real projective space, respectively, and $s_{0}$ and $p_{0}$ are arbitrary points. $\Sigma^{0}$ is sometimes denoted by $\overline{1}$ since it is the unit for the multiplication $\wedge$.

By $p(t, X, A)$ we denote the Poincaré polynomial of a pair $(X, A)$, i.e. the formal series $\sum_{k=0}^{\infty} \beta_{k} t^{k}$, where $\beta_{k}$ denotes the dimension of the $k$-th singular homology vector space with rational coefficients $H_{k}\left(X, A ; \mathbb{Q}\right.$ ) (we allow $\beta_{k}$ to be equal to $\infty$ ). If the Poincaré polynomial $p(t, X, A)$ is a polynomial in the usual sense then the Euler-Poincaré characteristic of $(X, A)$ is defined as

$$
\chi(X, A):=p(-1, X, A) .
$$

By the homotopy invarance of homology we can define

$$
p\left(t,\left[X, x_{0}\right]\right):=p\left(t, X,\left\{x_{0}\right\}\right) .
$$

In particular, $p\left(t, \Sigma^{k}\right)=t^{k}$ for every $k$ and $p\left(t, \Pi^{k}\right)=0$ provided $k$ is even, and $p\left(t, \Pi^{k}\right)=$ $t^{k}$ provided $k$ is odd. We also have

$$
\begin{aligned}
& p\left(t,\left[X, x_{0}\right] \vee\left[Y, y_{0}\right]\right)=p\left(t,\left[X, x_{0}\right]\right)+p\left(t,\left[Y, y_{0}\right]\right), \\
& p\left(t,\left[X, x_{0}\right] \wedge\left[Y, y_{0}\right]\right)=p\left(t,\left[X, x_{0}\right]\right) p\left(t,\left[Y, y_{0}\right]\right)
\end{aligned}
$$

(in the latter equation we assume that $X$ and $Y$ are CW-complexes).

Assume that $X$ is an ENR (Euclidean neighborhood retract). Let $f: X \rightarrow X$ be a continuous map and put

$$
\text { Fix } f:=\{x \in X: f(x)=x\} .
$$

A set $P$ is called an isolated set of fixed points of $f$ if it is a compact and open subset of Fix $f$. If $Q$ is an isolated set of fixed points of $g: X \rightarrow X$, we write $(f, P) \simeq(g, Q)$ 
provided there exists a continuous map $F: X \times[0,1] \rightarrow X \times[0,1], F(x, t)=\left(f_{t}(x), t\right)$ for some maps $f_{t}$ and an isolated set $P^{*}$ of fixed points of $F$ such that $f_{0}=f, f_{1}=g$, $P=\left\{x:(x, 0) \in P^{*}\right\}$, and $Q=\left\{x:(x, 1) \in P^{*}\right\}$.

A number $\operatorname{ind}(f, P) \in \mathbb{Z}$, called the fixed point index, is associated to an isolated set of fixed points $P$ of $f$ (see [Do]; we slightly changed the notation from that book in order to be consistent with the notation used in the Conley index theory). Below we recall some of its properties:

(i) $\operatorname{ind}(f, \emptyset)=0$.

(ii) If $P$ and $Q$ are isolated sets of fixed points of $f$ and $P \cap Q=\emptyset$ then $\operatorname{ind}(f, P \cup Q)=$ $\operatorname{ind}(f, P)+\operatorname{ind}(f, Q)$.

(iii) If $f: X \rightarrow X$ and $g: Y \rightarrow Y, P$ and $Q$ are isolated sets of fixed points of $f$ and $g$, respectively, then ind $(f \times g, P \times Q)=\operatorname{ind}(f, P) \operatorname{ind}(g, Q)$.

(iv) If $(f, P) \simeq(g, Q)$ then $\operatorname{ind}(f, P)=\operatorname{ind}(g, Q)$.

There are other important properties of the fixed point index, however we do not list them here.

3. The Conley index. Let $X$ be a metrizable locally compact space and let $\phi$ be a flow in $X$, i.e. a contiuous map

$$
\phi: X \times \mathbb{R} \ni(x, t) \rightarrow \phi_{t}(x) \in X
$$

such that $\phi_{0}=\operatorname{id}_{X}$ and $\phi_{s} \circ \phi_{t}=\phi_{s+t}$. For an arbitrary set $A \subset X$ put

$$
\begin{aligned}
\operatorname{Inv} A & :=\left\{x \in A: \forall t \in \mathbb{R}: \phi_{t}(x) \in A\right\}, \\
A^{-} & :=\left\{x \in A: \exists\left\{\epsilon_{n}\right\}, 0<\epsilon_{n} \rightarrow 0: \phi_{\epsilon_{n}}(x) \notin A\right\} .
\end{aligned}
$$

A compact set $S \subset X$ is called an isolated invariant set provided there exists $U$, a neighborhood of $S$, such that $S=\operatorname{Inv} U$. Such a $U$ is called an isolating neighborhood. A compact set $B \subset X$ is called an isolating block if $B^{-}$is also compact and $\operatorname{Inv} B \subset \operatorname{int} B$. The two basic results for the theory are:

THEOREM 1. If $U$ is an isolating neighborhood of $S$ then there exists an isolating block $B$ such that $S=\operatorname{Inv} B$ and $B \subset U$.

THEOREM 2. If $B_{1}$ and $B_{2}$ are isolating blocks such that Inv $B_{1}=\operatorname{Inv} B_{2}$ then

$$
\left[B_{1} / B_{1}^{-},\left[B_{1}^{-}\right]\right]=\left[B_{2} / B_{2}^{-},\left[B_{2}^{-}\right]\right] .
$$

It follows by Theorems 1 and 2 that if $S$ is an isolated invariant set then the pointed homotopy type

$$
h(\phi, S):=\left[B / B^{-},\left[B^{-}\right]\right],
$$

where $B$ is any isolating block such that $S=\operatorname{Inv} B$, is correctly defined. We call it the Conley index of $S$. (Frequently, in the definition of the index the notion of index pair is used. Each pair $\left(B, B^{-}\right)$, where $B$ is an isolating block is an example of index pair.) Before we list properties of the Conley index, we fix the following notation. Let $S$ and $T$ be isolated invariant sets for the flows $\phi$ and $\psi$, respectively, on the phase space $X$. We write $(\phi, S) \simeq(\psi, T)$ provided there exists a flow $\Phi$ on $X \times[0,1]$, an isolated invariant set $S^{*}$ for $\Phi$, and flows $\phi^{\sigma}$ on $X, \sigma \in[0,1]$ such that $\Phi_{t}(x, \sigma)=\left(\phi_{t}^{\sigma}(x), \sigma\right), \phi_{0}=\phi, \phi_{1}=\psi$, 
$S=\left\{x:(x, 0) \in S^{*}\right\}$, and $T=\left\{x:(x, 1) \in S^{*}\right\}$. If $\psi$ is a flow on a space $Y$, we define the flow $\phi \# \psi$ on $X \times Y$ by $(\phi \# \psi)_{t}(x, y):=\left(\phi_{t}(x), \psi_{t}(y)\right)$. Properties of the Conley index resemble the ones of the fixed point index:

(I) $h(\phi, \emptyset)=\overline{0}$

(II) If $S$ and $T$ are isolated invariant sets for $\phi$ and $S \cap T=\emptyset$ then $h(\phi, S \cup T)=$ $h(\phi, S) \vee h(\phi, T)$.

(III) If $S$ and $T$ are isolated invariant sets for flows $\phi$ on $X$ and $\psi$ on $Y$, respectively, then $h(\phi \# \psi, S \times T)=h(\phi, S) \wedge h(\psi, T)$.

(IV) If $(\phi, S) \simeq(\psi, T)$ then $h(\phi, S)=h(\psi, T)$.

Actually, there is a formula linking both indices (see [Mr1, Sr]):

Proposition 3. Assume that $S$ is an isolated invariant set of a flow $\phi$ on an ENRspace $X$. Put $S_{t}:=\left\{x \in S: \phi_{t}(x)=x\right\}$. Then, for every $t>0, S_{t}$ is an isolated set of fixed points of $\phi_{t}$ and

$$
\operatorname{ind}\left(\phi_{t}, S_{t}\right)=\chi(h(\phi, S)) .
$$

In particular, if $\chi(h(\phi, S)) \neq 0$ then there is at least one stationary point in $S$. In the case of a smoth flow the above formula is given in [Mc]. The Conley index provides some information on the topology of the isolated invariant set. Such information can be deduced from a long exact sequence from $[\mathrm{Ch}]$ and from results on Lusternik-Schnirelman type categories in $[\mathrm{Po}]$.

In the remainder of this section we assume that $X$ is a smooth manifold and $\phi$ is generated by the equation

$$
\dot{x}=f(x)
$$

where $f: X \rightarrow T X$ is a $C^{1}$-vector-field. Let $x_{0} \in X$ be a stationary point of $\phi$ (which means $f\left(x_{0}\right)=0$ ). We call it hyperbolic of index $k$ if $d_{x_{0}} f$ does not have eigenvalues on the imaginary axis and there are exactly $k$ eigenvalues (counted with multiplicities) with positive real part.

Proposition 4. Assume that $x_{0}$ is hyperbolic of index $k$. Then $\left\{x_{0}\right\}$ is an isolated invariant set and

$$
h\left(\phi,\left\{x_{0}\right\}\right)=\Sigma^{k} .
$$

Let $\gamma$ be a nontrivial periodic orbit of $\phi$. It is called hyperbolic of index $k$ if for some (equaivalently: any) point $x \in \gamma$ the diferential at $x$ of the Poincaré map of a section of $\gamma$ through $x$ does not have eigenvalues in the unit circle and there are exactly $k$ eigenvalues (counted with multiplicities) outside of the unit disc. Then the unstable manifold $W^{u}(\gamma)$ of $\gamma$ is of dimension $k+1$ and we call $\gamma$ untwisted (twisted) provided $W^{u}(\gamma)$ is orientable (nonorientable, respectively).

Proposition 5. Assume that $\gamma$ is hyperbolic of index $k$. Then $\gamma$ is an isolated invariant set and

(a) if $k=0$ then $h(\phi, \gamma)=\left[S^{1} \cup\{*\}, *\right]$,

(b) if $k \geq 1$ and $\gamma$ is untwisted then $h(\phi, \gamma)=\Sigma^{k} \vee \Sigma^{k+1}$,

(c) if $k \geq 1$ and $\gamma$ is twisted then $h(\phi, \gamma)=\Pi^{2} \wedge \Sigma^{k-1}$.

(One can check that $\left[S^{1} \cup\{*\}, *\right]$ is not the same as $\Sigma^{0} \vee \Sigma^{1}$.) 
4. Morse decompositions. Some ideas in the Conley index theory are derived from research on critical points of functionals. Let $X$ be a Riemannian manifold of dimension $n$ and let $f: X \rightarrow \mathbb{R}$ be a $C^{1}$ map with locally Lipschitzean gradient $\nabla f$. The equation

$$
\dot{x}=-\nabla f(x)
$$

induces a (local) flow $\phi$ on $X$. Critical points of $f$ (i.e. points such that the derivative of $f$ is zero) coincide with stationary points of $\phi$. Moreover, if $x_{0}$ is an isolated critical point of $f$ (i.e. the unique critical point in some of its neighborhood) then $\left\{x_{0}\right\}$ is an isolated invariant set for $\phi$. Assume additionally that $f$ is of $C^{2}$ class. A critical point $x_{0}$ is called nondegenerate if the Hessian of $f$ at $x_{0}$ is a nondegenerate quadratic form. There exists a canonical base in which the Hessian has a diagonal form with $k$ negative and $n-k$ positive entries. The number $k$ is an invariant of the nondegenerate critical point and is called its Morse index. The Morse Lemma asserts that if $x_{0}$ is a nondegenerate critical point of index $k$ then there exists a chart $g: U \rightarrow \mathbb{R}^{n}$ with $g\left(x_{0}\right)=0$ such that

$$
f\left(g^{-1}(v)\right)=f\left(x_{0}\right)-\sum_{i=1}^{k}\left(v^{i}\right)^{2}+\sum_{i=k+1}^{n}\left(v^{i}\right)^{2},
$$

where $v=\left(v^{1}, \ldots, v^{n}\right) \in g(U)$. Since each nondegenerate critical point is isolated, the Conley index of $\left\{x_{0}\right\}$ is defined and, by (1),

$$
h\left(\phi,\left\{x_{0}\right\}\right)=\Sigma^{k} .
$$

Thus the Conley index directly generalizes the Morse index — in particular, it applies to arbitrary isolated critical points, not to nondegenerate ones only.

It is natural to look for further results on the Conley index which are motivated by Morse theory. One of ideas comes from the observation that critical points are naturally ordered by their $f$-values. This implies some restrictions on possible trajectories connecting them - they must go from points greater to points less in that order, which leads to a general rule of endowing an order relation in a collection of disjoint invariant subsets of an isolated invariant set. More exactly, let $\phi$ be a flow on a metrizable and locally compact space $X$ and let $S$ be an isolated invariant set for $\phi$. Assume that $\mathcal{M}$ is a finite set of disjoint compact invariant subsets of $S$. A (partial) order relation $\leq$ in $\mathcal{M}$ is called admissible if for every $x \in S \backslash \bigcup \mathcal{M}$ there are $M<N$ in $\mathcal{M}$ such that $\alpha(x) \subset N$ and $\omega(x) \subset M$. The set $\mathcal{M}$ endowed with an admissible order is called a Morse decomposition and its elements are called Morse sets (see [C2, Smo]). In particular, each Morse set is an isolated invariant set for $\phi$. In the following result we present a formula on Poincaré polynomials of Morse sets. Traditionally it is called "Morse inequalities".

THEOREM 6 (Morse inequalities). If $S$ is an isolated invariant set for the flow $\phi$ and $\mathcal{M}$ is its Morse decomposition then

$$
\sum_{M \in \mathcal{M}} p(t, h(\phi, M))=p(t, h(\phi, S))+(1+t) Q(t),
$$

where $Q(t)$ is a formal series with coefficients being nonnegative integers or $\infty$.

The above formula generalizes the classical Morse inequalities due to Morse (for Morse functions) and to Smale (for Morse-Smale flows, see [Sma]). Indeed, let $X$ be a compact 
smooth $n$-dimensional manifold, $\phi$ be a smooth flow on $X$ and assume that the chain recurrent set consists of hyperbolic stationary points and hyperbolic periodic orbits only. It follows that the stationary points and periodic orbits form the Morse decomposition of $X$. Denote by $\beta_{k}$ the $k$-th Betti number of $X$, by $c_{k}$ the number of stationary points of index $k$, and by $A_{k}$ the number of untwisted periodic orbits. Since the Poincaré polynomial of $\Pi^{2} \wedge \Sigma^{k-1}$ is equal to 0 , twisted periodic orbits do not appear in (2). Thus, there are terms $\beta_{k} t^{k}, c_{k} t^{k}$, and $A_{k} t^{k}+A_{k} t^{k+1}$ in the formula (2). By the equation $(1+t)^{-1}=$ $1-t+t^{2}-\ldots$ and comparision of monomials one can deduce that

$$
c_{k}-c_{k-1}+\ldots \pm c_{0}+A_{k} \geq \beta_{k}-\beta_{k-1}+\ldots \pm \beta_{0},
$$

where $0 \leq k \leq n$. In particular,

$$
\sum_{i=0}^{n}(-1)^{i} c_{i}=\chi(X)
$$

\section{References}

[Ba] T. BARTSCH, Topological methods for variational problems with symmetries, Lecture Notes 1560, Springer-Verlag, Berlin, 1993,

[Be] V. BEnCI, A new approach to the Morse-Conley theory and some applications, Ann. Math. Pura Appl. 4 (1991), 231-305.

[Do] A. Dold, Lectures on Algebraic Topology, Springer-Verlag, Berlin, Heidelberg and New York, 1980.

[Ch] R. C. Churchill, Isolated invariant sets in compact metric spaces, J. Differential Equations 12 (1972), 330-352.

[C1] C. C. Conley, On a generalization of the Morse index, in: Ordinary Differential Equations, 1971 NRL-MRC Conference (L. Weiss, Ed.), Academic Press, New York and London, 1972, 133-146.

[C2] C. C. Conley, Isolated Invariant Sets and the Morse Index, CBMS Regional Conf. Ser. Math. 38, AMS, Providence, R.I., 1978.

[F1] R. Franzosa, The continuation theory for Morse decompositions and connection matrices, Trans. Amer. Math. Soc. 310 (1988), 781-803.

[F2] R. Franzosa, The connection matrix theory for Morse decompositions, Trans. Amer. Math. Soc. 311 (1989), 561-592.

[FM] R. Franzosa and K. Mischaikow, The connection matrix theory for semiflows on (not necessarily locally compact) metric spaces, J. Differential Equations 71 (1988), $270-287$.

[Ge] K. GȨBA, Degree for gradient equivariant maps and equivariant Conley index, Topological nonlinear analysis, II (Frascati, 1995), 247-272, Progr. Nonlinear Differential Equations Appl. 27, Birkhäuser, Boston, 1997.

[KM] T. KaCZynski and M. MrozeK, Conley index for discrete multi-valued dynamical systems, Topology Appl. 65 (1995), 83-96.

$[\mathrm{Ku}] \mathrm{H}$. L. KuRLAND, The Morse index of an isolated invariant set is a connected simple system, J. Differential Equations 42 (1981), 234-259.

[Mc] C. McCord, On the Hopf index and the Conley index, Trans. Amer. Math. Soc. 313 (1989), 853-860. 
[McM] C. MCCORD and K. Mischaikow, Connected simple systems, transition matrices, and heteroclinic bifurcations, Trans. Amer. Math. Soc. 333 (1992), 379-422.

[Mi1] K. Mischaikow, Transition systems, Proc. Roy. Soc. Edinburgh, Sect. A 112 (1989), $155-175$.

[Mi2] K. Mischaikow, Conley index theory, in: Dynamical Systems, CIME-Session, Montecatini Terme 1994, 119-207, Lecture Notes 1609, Springer-Verlag, Berlin, 1995.

[Moe] R. MoEckel, Morse decompositions and connection matrices, Ergodic Theory Dynamical Systems 8* (1988), 227-249.

[Mon] J. T. Montgomery, Cohomology of isolated invariant sets under perturbation, J. Differential Equations 13 (1973), 257-299.

[Mr1] M. Mrozek, Periodic and stationary trajectories of flows and ordinary differential equations, Univ. Iagel. Acta Math. 27 (1988), 29-37.

[Mr2] M. MrozeK, A cohomological index of Conley type for multivalued admissible flows, J. Differential Equations 84 (1990), 15-51.

[Mr3] M. MrozeK, Leray functor and cohomological index for discrete dynamical systems, Trans. Amer. Math. Soc. 318 (1990), 149-178.

[Po] M. PoŹNIAK, Lusternik-Schnirelman category of an isolated invariant set, Univ. Iagel. Acta Math. 31 (1994), 129-139.

[Re] J. F. REINECK, The connection matrix in Morse-Smale flows, Trans. Amer. Math. Soc. 322 (1990), 523-545.

[RS] J. Robbin and D. Salamon, Dynamical systems, shape theory and the Conley index, Ergodic Theory Dynamical Systems 8* (1988), 375-393.

[Ry] K. P. Rybakowski, The Homotopy Index and Partial Differential Equations, SpringerVerlag, Berlin, Heidelberg and New York, 1987.

[S1] D. Salamon, Connected simple systems and the Conley index of isolated invariant sets, Trans. Amer. Math. Soc. 291 (1985), 1-41.

[S2] D. Salamon, Morse theory, the Conley index and Floer homology, Bull. London Math. Soc. 22 (1990), 113-140.

[Sma] S. Smale, Morse inequalities for a dynamical system, Bull. Amer. Math. Soc. 66 (1960), 43-49.

[Smo] J. Smoller, Shock Waves and Reaction-Diffusion Equations, Springer-Verlag, New York and Berlin, 1983.

[Sr] R. SRzednicki, Periodic and bounded solutions in blocks for time-periodic nonautonomous ordinary differential equations, Nonlinear Anal. — Theory Meth. Appl. 22 (1994), 707-737.

[Sz] A. SzymCZAK, The Conley index for discrete semidynamical systems, Topology Appl. 66 (1995), 215-240. 